\title{
Adsorption and De-Sorption of Polycyclic Aromatic Hydrocarbons on Activated Carbon
}

\author{
Changming Zhang ${ }^{1 *}$, Xiaohang Zhang ${ }^{1}$, Zhanggen Huang ${ }^{1}$, Dongmei Huang ${ }^{1}$ and Qiuxia Cheng ${ }^{2}$ \\ ${ }^{1}$ State Key Laboratory of Coal Conversion, Institute of Coal Chemistry, Chinese Academy of Sciences, Taiyuan 030001, China \\ ${ }^{2}$ Shanxi Northern Xingan Chemical Industry Co.Ltd, China
}

\begin{abstract}
The adsorption and de-sorption properties of polycyclic aromatic hydrocarbons (PAH) which was represented by pure phenanthrene, on activated carbon were investigated. The influences of solvent (acetone,carbon tetrachloride, tetrahydrofuran, ethanol or distilled water), time and manner (with ultrasonic or not) on the desorption efficiency were investigated. The results show that the de-sorption efficiency reaches $91.40 \%$ which exhibits the highest efficiency when the ethanol is used as the de-sorption solvent and the system was treated with ultrasonic technology. The desorption test was also carried on the raw coconut shell-based activated carbon and it was not being used before. This determination is to understand the "baseline background" and the purity of raw activated carbon characteristics. The content of total de-sorption amounts was $0.043 \%$ of raw activated carbon. Through GC/MS analysis,the main desorption substance defined was 10-undecenoic acid methyl ester.
\end{abstract}

Keywords: Activated carbon; Adsorption; De-sorption; Phenanthrene; GC / MS

\section{Introduction}

Most of the active carbon is black powder or granular amorphous carbon. In addition to its main component carbon, it also contains oxygen, hydrogen and other elements. Activated carbon has large specific surface area and good adsorption characteristics. Activated carbon is often used for water purification including tap water, industrial water, wastewater and pharmaceutical water, gas adsorption, such as air purification, gas purification, and harmful emissions control. In addition, activated carbon is also used in some high demand areas, such as blood purification [1], recovery of precious metals like gold and silver [2] and making high-performance fuel cells [3,4]. Activated carbon almost relates with all industrial sectors of people's daily lives.

With the rapid development of activated carbon industry, the study on adsorption and/or desorption of activated carbon increases rapidly. In literatures, there are many reports about adsorption properties of activated carbon, which include the adsorptions of many compounds and metals, such as methyl tertiary-butyl ether, trichloroethene [5], resorcinol, catechol [6], mercury [7,8], phenanthrene [9], p-nitrophenol [10], naphthalenesulphonic acids [11], aromatics [12], Hg-Ni-Cd metal ions [13] etc. However the report about de-sorption of activated carbon is less [14].

The de-sorption performance of activated carbon is one important aspect of the properties of activated carbon. To research de-sorption of activated carbon is indispensable to fully understand essentials of activated carbon. By application of de-sorption, it can solve the identification of specific substances, which were adsorbed on activated carbon, and provide the necessary information for some specific areas, such as blood purification, food additives and other deep-level activated carbon application.

This study is also relevant with issues of environmental protection. People in modern life give great attention to air purification, especially to purification of industrial emissions. Currently, activated carbon impregnated with catalyst has been used in desulfurization and denitrification [15].

However, the often gas in industrial emissions contain levels of
PAH. Analogously with desulfurization and denitrification processes, key questions to answer are how will the PAH impact to them when carried out by applying carbon-base catalyst? When activated carbon is regenerated, how will the PAH affect the regeneration efficacy? Some PAHs are also carcinogens, and handling of these chemicals requires safety protocols? These environmental issues need to be researched.

According to above needs about deep-level activated carbon application and environmental protection, an exploratory study was made. Firstly, our study focused on adsorption and de-sorption of $\mathrm{PAH}$, especially on the de-sorption characteristics. Through a series of experiments, results obtained show some significance.

\section{Experimental Procedure}

\section{Apparatus, reagents and samples}

The GC-MS instrument used was a Finnigan tracking GC-MS Analyzer (U.S. Finnigan Corporation).

The CSF-1A ultrasonic generator was a model from Shanghai (City, China) Ultrasonic Instrument Factory. The applied current was at $200 \mathrm{~mA}$.

The spectrophotometer 752 UV was a model from Shanghai Analytical Instrument Factory.

The chloroform, carbon tetrachloride, tetrahydrofuran, ethanol and methanol were of analytical grade reagents from Tianjin Bodi Chemical Co., Ltd. production.

${ }^{*}$ Corresponding author: Changming Zhang, State Key Laboratory of Coal Conversion, Institute of Coal Chemistry, Chinese Academy of Sciences, Taiyuan 030001, China, Tel: +86-351-4043727; Fax: +86-351-4043727; E-mail: zhangcm@ sxicc.ac.cn

Received December 09, 2011; Accepted January 03, 2012; Published January 05, 2012

Citation: Zhang C, Zhang X, Huang Z, Huang D, Cheng Q (2012) Adsorption and De-Sorption of Polycyclic Aromatic Hydrocarbons on Activated Carbon. J Environment Analytic Toxicol 1:116. doi:10.4172/2161-0525.1000116

Copyright: $\odot 2012$ Zhang C, et al. This is an open-access article distributed unde the terms of the Creative Commons Attribution License, which permits unrestricted use, distribution, and reproduction in any medium, provided the original author and source are credited. 
Coconut-shell activated carbon was provided by Coconut-Island Environmental Protection Technology Co., Ltd. of Zhengzhou (Zhengzhou, city in China).

\section{Experimental method and operation}

Preparation of activated carbon: Through experimental and taking all factors considered into account, the optimum operation conditions were defined as follows.

Phenanthrene $0.0100 \mathrm{~g}$ was accurately weighed and placed into a $200 \mathrm{ml}$ flask, the $100 \mathrm{ml}$ hexane was added in to the flask. The solution was shaken for 10 minutes to fully dissolve the phenanthrene. The solution was placed for 20 minutes, and then its UV absorbance was measured by UV spectrophotometer.

Subsequently, the activated carbon of $0.5000 \mathrm{~g}$ was placed into the above flask. The flask was shaken for 10 minutes and then stored for 1 hour for filtration/adsorption (at room temperature). After adsorption, the clear liquid in flask was extracted; and its absorption determined by UV spectrophotometer.

The residual activated carbon, in which activated carbon had adsorbed phenanthrene, was placed into a vacuum dryer under nitrogen atmosphere at $25^{\circ} \mathrm{C}$. In dryer under hexane was constantly eluted out, until activated carbon was the constant weight. And thus, the activated carbon was completely loaded with phenanthrene.

\section{De-sorption of phenanthrene from activated carbon}

Effects of different solvents on the de-sorption efficiency: The $0.5000 \mathrm{~g}$ of activated carbon with adsorbed phenanthrene was weighed and placed into the flask, subsequently $100 \mathrm{ml}$ of solvent was added. The solution was shaken for 1 hour and then stored for 1 hour at room temperature. The solution of $100 \mathrm{ml}$ was taken out and measured by UV. The residual solution in the flask was dissolved with $100 \mathrm{ml}$ solvent, then shaken for 1 hour and kept at rest for 1 hour, at room temperature. The above operation was repeated 5 times until a total solvent of $600 \mathrm{ml}$ were used.

The different solvents including acetone, carbon tetrachloride, tetrahydrofuran, ethanol, distilled water were used, then the different de-sorption effects were determined.

The calculation formula of the de-sorption efficiency was as follows:

$$
\mathrm{Des}_{\text {ef }}(\mathrm{W} \%)=\mathrm{ABS}_{\text {end }} / \mathrm{ABS}_{\text {or }}{ }^{*} 100 \%
$$

In which Des ${ }_{\text {ef }}$ is the de-sorption efficiency; $\mathrm{ABS}_{\text {end }}$ and $\mathrm{ABS}_{\text {or }}$ are $\mathrm{UV}$ absorption values of final de-sorption solution and the solution of original phenanthrene, respectively.

It should be noted that the "original solution" is the solution corresponding to $0.0100 \mathrm{~g}$ phenanthrene used in preparation of activated carbon (adsorbing phenanthrene).

Effects of de-sorption time and manner on the de-sorption efficiency: Four activated carbons lots were prepared (paragraph 4.2.1) for de-sorption experiments, where each amount of activated carbon was loaded with same amounts of phenanthrene.

The experiments operation of de-sorption were the same as that described by previous section, and the total amount used solvent of every activated carbon was $1000 \mathrm{ml}$. One of the four runs was used with ultrasonic treatment; the ultrasound operation was repeated for 8 times every 0.5 hour.

De-sorption of fresh activated carbon: The fresh activated carbon is coconut shell type has never been used. In the de-sorption process, the sample amount is 20 grams and the de-sorption procedure was the same as the previous paragraph.

The procedure of using of ultrasonic vibration was repeated for 9 times. The total amount of solvent was $1000 \mathrm{ml}$. Finally, the solution was filtrated, and then remaining solution was placed in a vacuum system to remove the solvent. The residues (de-sorption substances) were analyzed by GC / MS.

The optimal analysis conditions of GC/MS: column was a DB5MS, 30 meters long, $0.25 \mathrm{~mm}$ diameter, and $0.25 \mathrm{~mm}$ film thickness. Carrier gas helium of $1.0 \mathrm{ml} / \mathrm{min}$, the initial temperature of $70^{\circ} \mathrm{C}$ for 3 minutes, then $10^{\circ} \mathrm{C} / \mathrm{min}$ up rate, and finally heated to $250^{\circ} \mathrm{C}$ for 3 minutes. Electron impact was set at 70 electron volts $(\mathrm{eV})$, atomic mass (amu) range was 30-350.

\section{Results and Discussion}

\section{Adsorption efficiency of activated carbon with phenanthrene}

By comparing the UV absorption of the original solution and the solution after adding activated carbon, the adsorption efficiency can be determined. The results of adsorption efficiency are listed in Table 1 . With 5 runs as statistical base, the average adsorption efficiency was deduced to $99.91 \%$, and the average value of adsorption capacity of AC is 20.22 ( $\mathrm{mg} / \mathrm{g}$ phenanthrene).

The result indicates that activated carbon has high adsorption ability, and phenanthrene can be easily loaded on activated carbon. Some related mechanisms were described simply as follows.

In $\mathrm{PAH}$ phenanthrene molecule, there are $\pi$ bonds; While in there are $\pi$-bonds between carbon layers and there are carbonyl carbon, quinone-based, acid hydroxyl and other polar groups in carbon molecule $[16,17]$.

When carbon molecules contact with phenanthrene molecular,

\begin{tabular}{|c|c|c|c|c|c|}
\hline No & $\begin{array}{l}\text { Concentration } \\
\text { of phenanthrene } \\
\mathrm{g} / 100 \mathrm{ml}\end{array}$ & $\begin{array}{l}\text { Absorption } \\
\text { of solution } 1 \\
\text { (UV } 270 \mathrm{~nm})\end{array}$ & $\begin{array}{l}\text { Absorption } \\
\text { of solution } 2 \\
\text { (UV 270nm }\end{array}$ & $\begin{array}{l}\text { Adsorption efficiency } \\
\%\end{array}$ & $\begin{array}{l}\text { Adsorption capacity of } \\
\text { AC } \mathrm{mg} / \mathrm{g}\end{array}$ \\
\hline 1 & 0.0100 & 0.9928 & 0.0012 & 99.879 & 19.975 \\
\hline 2 & 0.0102 & 0.9937 & 0.0011 & 99.889 & 20.377 \\
\hline 3 & 0.0101 & 0.9925 & 0.0007 & 99.929 & 20.185 \\
\hline 4 & 0.0100 & 0.9927 & 0.0006 & 99.939 & 19.987 \\
\hline 5 & 0.0103 & 0.9931 & 0.0008 & 99.919 & 20.583 \\
\hline Average & 0.0101 & 0.9930 & 0.0009 & 99.911 & 20.222 \\
\hline
\end{tabular}

Table 1: Adsorption efficiency of phenanthrene on activated carbon 
between $\pi$ bonds, instantaneous dipole will be produced and further dispersion force will be produced. Polar molecule phenanthrene has "inherent dipole" being produce dipole moment forcer. In addition, through electric field of "intrinsic dipole", other polar and non polar molecules often produce new dipole named with as "induced dipole". The induced force would be generated from "induced dipole".

Above referred forces, which are including dispersion, induced force and dipole force, are not negligible, especially force derived from dipole. When two polar molecules (Philippines and activated carbon) close, the dipole will work. If poles are same then they would exclude each other; if two poles are different pole then they would attract each other. As different poles continually close to, two molecules continually also close to, finally this close to certain distance, the repulsion and gravitational achieve equilibrium and adsorption process is completed.

Due to Phenanthrene and activated carbon provide many dipoles and electron motion is fast, so the phenanthrene was easily and strongly adsorbed on carbon. And often non-pole solute can not to reach these.

\section{De-Sorption of Phenanthrene on Activated Carbon}

Effects of different solvents on the de-sorption efficiency: The different solvents including acetone, carbon tetrachloride, tetrahydrofuran, ethanol, distilled water were used at the same operation conditions, and with a total volume of $600 \mathrm{ml}$ of solvent liquid, without ultrasound. The effects of different solvents on the desorption efficiency are shown in Figure 1.

To complete de-sorption of the phenanthrene, three factors are needed. The first, the solvent has capable to good solubility to phenanthrene; Second, the solvent molecules is smaller and then it easy penetrate and deep into the porous of carbon; Third, if polar functional groups $(\mathrm{O}=,-\mathrm{O}-,-\mathrm{OH})$ has a high proportion in solvent, the de-sorption effect is more dominant. In the five de-sorption agent, the dissolved degree of aromatics in water is the worst of, therefore, the de-sorption efficiency is minimum with water.

Through handbooks and calculations, some basic data would be getting about other four organic solvents. The molecular weight of ethanol, acetone, tetrahydrofuran, carbon tetrachloride is successively $46.07,58.08,75.11$, and 153.84 and their proportion of polar functional groups is successively $36.90,27.55,22.19,0$ (W\%).

In our examples, de-sorption efficiencies of ethanol, acetone, tetrahydrofuran, carbon tetrachloride are successively are successively $40.011,32.327,11.942,5.32$

The changes characteristics of above three sets clearly reveal that the relationship of de-sorption effect of phenanthrene and property of solvent used follow a stringent law.

Effects of de-sorption time and manner : With the same desorption procedure (as mentioned in chapter 5.2.1), the $0.5000 \mathrm{~g}$ of activated carbon with adsorbed phenanthrene of run1, run2 and run3 was weighed and placed into the flask, subsequently $100 \mathrm{ml}$ of solvent was added. The solution was shaken for 1 hour and then stored for 1 hour (at room temperature). The solution was taken out and measured by UV. The residual solution in the flask was dissolved with $100 \mathrm{ml}$ solvent. The above operation was repeated 9 times until a total solvent of $1000 \mathrm{ml}$ were used. Every de-sorption efficiency was get from UV determination. The ultrasonic treatment was used in one (run4). The relations of de-sorption efficiency with the amount of solvent and ultrasonic are shown in Figure 2. In Figure $\mathrm{X}$-axis represent $\mathrm{ml}$ amount of solvent used and Y-axis represent the recover rate namely de-sorption efficiency.

The results (Figure 2) clearly show that the de-sorption efficiency increases with the amount solvent. When ultrasonic treatment is used, the de-sorption efficiency is much higher than that of without ultrasonic. The maximum de-sorption efficiency is $91.40 \%$. In addition, the de-sorption rate increased with increasing amount solvent used, however, after $900 \mathrm{ml}$ used then showed a tendency of balance. The maximum volume applicable to maximum elution seems therefore to reach $900 \mathrm{ml}$ for the given amount of activated carbon.

After considering the results above and by taking all factors into account, the optimum process conditions were finally defined as follows: The amount of activated carbon: $0.5000 \mathrm{~g}$. The solvent used is ethanol, the total amount solvent used is $900 \mathrm{ml}$, and the ultrasonic treatment is used. This gives a desorption efficiency of $>90 \%$.

These experimental results also deeply imply that adsorption and de-sorption are reversible, but adsorption yields better efficiency than de-sorption; it is also the main reason that activated carbon is usually used as adsorbent.

\section{De-Sorption of fresh activated carbon}

The average content of total de-sorption of fresh active carbon is

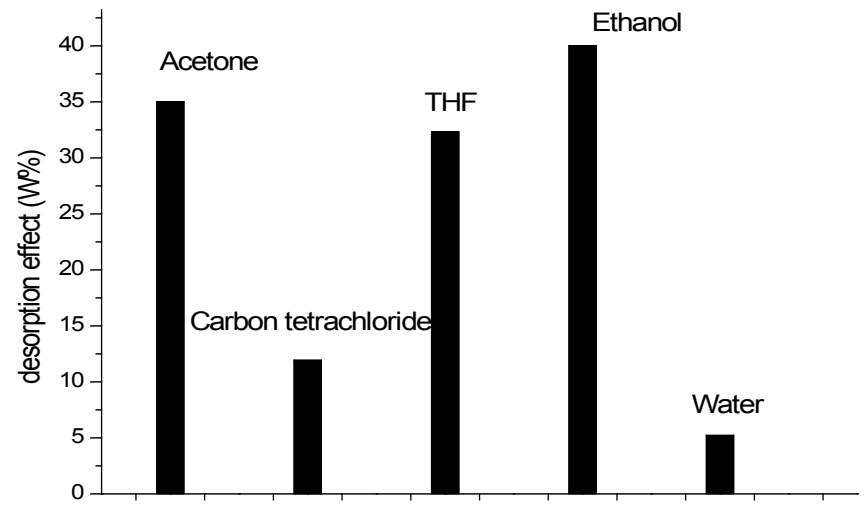

Figure 1: Comparison of de-sorption effect using different solvents.

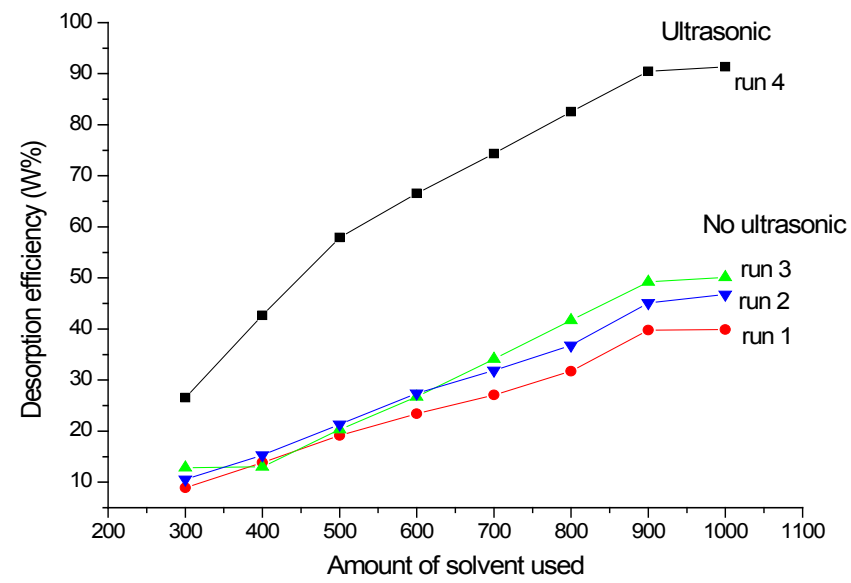

Figure 2: Effect of solvent amount and ultrasound on de-sorption. 
Citation: Zhang C, Zhang X, Huang Z, Huang D, Cheng Q (2012) Adsorption and De-Sorption of Polycyclic Aromatic Hydrocarbons on Activated Carbon. J Environment Analytic Toxicol 1:116. doi:10.4172/2161-0525.1000116

measured to $0.043 \%$ (W \%). It should be noted that an average value is obtained by 3 runs. By GC/MS analysis of de-sorption of active carbon, the total flow ion chromatogram of de-sorbed substances is shown in Figure 3. Typical MS qualitative comparison $(\mathrm{m} / \mathrm{z})$ is shown in Figure 4.

By qualitative analysis, the main organic extractions of de-sorption is 10-undecenoic acid methyl ester. It is clearly seen that the $\mathrm{m} / \mathrm{z}$ of the chief de-sorption substance from activated carbon at 11.30 minutes of total flow ion chromatogram is almost the same as that of pure 10-undecenoic acid methyl ester. The characteristic values of $\mathrm{m} / \mathrm{z}$ of pure 10-undecenoic acid methyl ester and the de-sorption substance

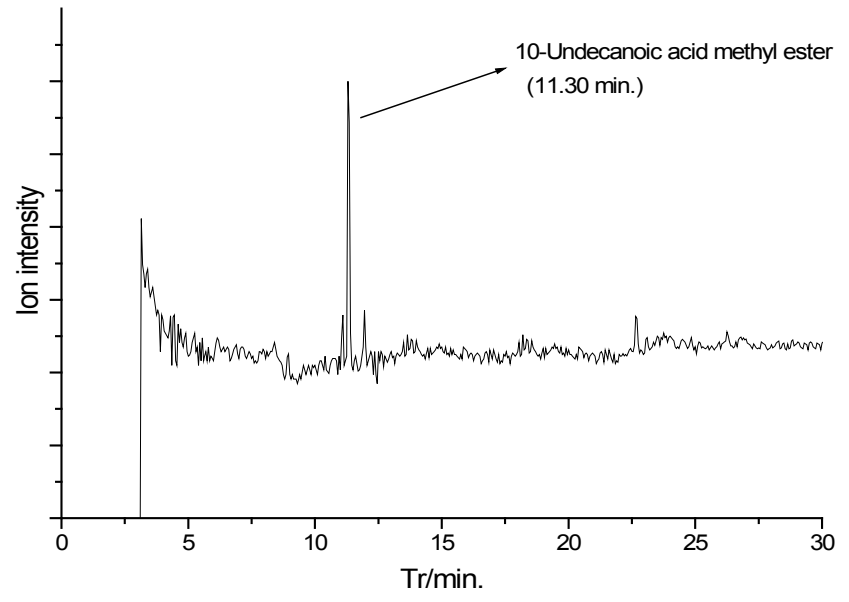

Note: The retention of composition (from AC) was 11.3min. (From Figure.3

Figure 3: The total flow ion chromatogram of de-sorption substance.

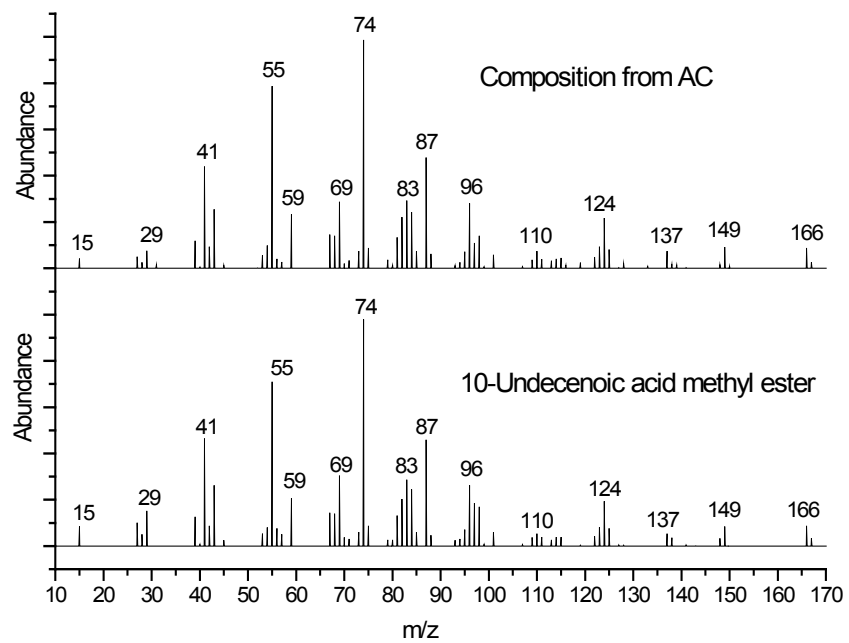

Figure 4: The $\mathrm{m} / \mathrm{z}$ comparison of de-sorption substance and pure reagent.<smiles>C=CCCCCCCCCC(=O)OC</smiles>

Figure 5: Structure of 10-undecenoic acid methyl ester. are mainly $15,29,41,55,59,69,74,83,87,96,110,124,149,166$. Thus the 10-undecenoic acid methyl ester in de-sorption substance is accurately defined with the qualitative matching of $93.3 \%$. The structure of 10 -undecenoic acid methyl ester is shown in Figure 5.

The results show also that the activated carbon is not absolute "pure" and nontoxic. These imply that activated carbon cannot be used as medicine or food additives.

\section{Conclusion}

A systematic study was carried out to characterize of adsorption and de-sorption of pure phenanthrene on activated carbon. The optimum de-sorption conditions are activated carbon $0.5000 \mathrm{~g}$ containing phenanthrene, ethanol as extraction solvent, the total used amount of $1000 \mathrm{ml}$, with the ultrasonic treatment. The average value of highest de-sorption efficiency is $91.40 \%$.

The results of de-sorption of fresh coconut shell-based activated carbon is that the average value of de-sorption efficiency is $0.043 \%$ ( $\mathrm{w} \%$ ), and through GC / MS analysis the main substance from desorption was confirmed as 10-Undecenoic acid methyl ester.

These results show that "fresh" activated carbon is not absolutely pure and potentially toxic, underlining that it cannot be used as medicine or food additives.

\section{Acknowledgments}

The authors gratefully acknowledge financial support from the National Natura Science Foundation of China (Nos. 20677065), Foundation of Natural Science Shanxi Province (Nos.2008011014-3).

\section{References}

1. Ma $Y$ (2006) Research progress about adsorbent for blood purification Chinese Journal of Blood Purification 5: 783-810.

2. Rosato LI (2002) The recovery of precious metals from zinc- leach- residue by applying thiourea leaching method. Science \& Technology of Zhuzhou Smelters 18: 29-33.

3. Yuban Tamg (2011) Direct carbon solid oxide fuel cell. A Dissertation Submitted for the Degree of Doctor of Philosophy of South China University of Technology 1-110

4. Joon K (1998) Fuel cells-a 21st century power system. Journal of Powe Sources 71: 12-18

5. Lei L, Quinlivan PA, Detlef RUK (2002) Effects of activated carbon surface chemistry and pore structure on the adsorption of organic contaminants from aqueous solution. Carbon 40: 2085-2100.

6. Arinjay K, Shashi K, Surendra K (2003) Adsorption of resorcinol and catecho on granular activated carbon: Equilibrium and kinetics. Carbon 41: 3015 3025 .

7. Li YH, Lee CW, Gullett BK (2002) The effect of activated carbon surface moisture on low temperature mercury adsorption. Carbon 40: 65-72.

8. Radisav DV, Douglas PS (2001) Vapor-phase elemental mercury adsorption by activated carbon impregnated with chloride and chelating agents. Carbon 39: 3-14.

9. Murillo R, García T, Aylón E, Callén MS, Navarro MV, et al. (2004) Adsorption of phenanthrene on activated carbons: Breakthrough curve modeling. Carbon 42: 2009-2017.

10. Haydar S, Ferro-Garc'ıa MA, Rivera-Utrilla J, Joly JP (2003) Adsorption of p-nitrophenol on an activated carbon with different oxidations. Carbon 41 387-395.

11. Rivera-Utrilla J Sa'nchez-PoloM(2002)The role of dispersive and electrostatic interactions in the aqueous phase adsorption of naphthalenesulphonic acids on ozone-treated activated carbons. Carbon 40: 2685-2691.

12. Leng CC, Pinto, NG (1997) Effects of surface properties of activated carbons on adsorption behavior of selected aromatics. Carbon 35: 1375-1385. 
Citation: Zhang C, Zhang X, Huang Z, Huang D, Cheng Q (2012) Adsorption and De-Sorption of Polycyclic Aromatic Hydrocarbons on Activated Carbon. J Environment Analytic Toxicol 1:116. doi:10.4172/2161-0525.1000116

Page 5 of 5

13. Dastgheib SA, Rockstraw DA (2002) A systematic study and proposed model of the adsorption of binary metal ion solutes in aqueous solution onto activated carbon produced from pecan shells. Carbon 40: 1853-1861.

14. Do DD, Junpirom SH (2009) A new adsorption-desorption model for water adsorption in activated carbon. Carbon 47: 1466-1473.

15. Jianrong $M$, Zhengu L, Shoujun L, Zhenping Z (2003) A regenerable Fe/AC desulfurizer for $\mathrm{SO}_{2}$ adsorption at low temperatures. Appl Catal B Environ 45: 301-309.

16. Li ZS, Zhang G, Li JX, Wang XL, Liu XQ (2009) The electrical properties of $\mathrm{PVC} / \mathrm{CB}$ negative temperature coefficient composites, Proceeding of the Ninth National Symposium on New Carbon Materials, China, 222-227.

17. Donnet JB (1982) Structure and reactivity of carbon: From carbon black to carbon composites. Carbon 20: 267-282. 